\title{
Controlled actuation of Nafion-based Ionic Polymer-metal Composites (IPMCs) with Ethylene Glycol as Solvent
}

\author{
Shahram Zamani and Sia Nemat-Nasser \\ University of California, San Diego \\ Center of Excellence for Advanced Materials \\ 9500 Gilman Drive \\ La Jolla, CA 92093-0416
}

\begin{abstract}
Ionic polymer-metal composites (IPMCs) consist of a perfluorinated ionomer membrane (usually Nafion ${ }^{\circledR}$ or Flemion ${ }^{\circledR}$ ). The ionomer is plated on both faces with a noble metal such as gold or platinum. It is neutralized with a certain amount of counterions that balance the electrical charge of anions covalently fixed to the backbone membrane. IPMCs are electroactive materials that can be used as actuators and sensors. Their electrical-chemical-mechanical response is highly dependent on the cations used, the nature and the amount of solvent uptake, the morphology of the electrodes, and other factors. When a cantilever strip of solvated Nafion-based IPMC sample is subjected to a suddenly applied and sustained (DC) electric potential of several volts (1-3 V) across its faces, it bends towards the anode. For Nafion-based IPMCs with alkali metals, actuation towards the anode is followed by a slow back relaxation towards the cathode. If the electric potential is removed and the two electrodes are shorted during this back relaxation, the sample displays a fast bending deformation towards the cathode and then slowly relaxes back towards the anode, attaining a new equilibrium position generally distinct from its initial state. One way to change various phases of IPMC actuation is achieved by changing input potential. The electric potential inputs may be used to control the actuation of IPMCs. We present the results of a series of tests on Nafion-based IPMCs with ethylene glycol as solvent, actuated under electric potential inputs other than DC electric potential. We present experimental results for increasing ramp and sinusoidal electric potential waveforms.
\end{abstract}

\section{Introduction}

Ionic polymer-metal composites (IPMCs) are electroactive materials with potential applications as actuators and sensors. When a thin strip of an IPMC membrane in the solvated state is subjected to a suddenly imposed and sustained constant electric potential (DC) of several volts (1-3 V), it bends towards the anode. For Nafion-based IPMCs that are neutralized with alkali metals, the strip then slowly relaxes back towards the cathode. This occurs while the sample is still under electric potential [Nemat-Nasser and Thomas, 2001 and Nemat-Nasser, 2002]. Experimental observations for Flemion-based IPMCs [Nemat-Nasser and Wu, 2003] show that the initial actuation towards the anode is followed by slower relaxation in the same (i.e., towards the anode) direction. Hence, unlike Nafion-based IPMCs, no back relaxation has been detected for Flemion-based IPMCs, in experiments conducted so far. Electrical-chemical-mechanical actuation of Nafion-based IPMCs upon application of applied electric potential depends on the morphology of backbone ionomer, type of incorporated cation [Onishi et al., 2001], type of solvent, degree of salvation, and morphology of the electrodes [Onishi et al., 2000].

In Nemat-Nasser micromechanical model for actuation of IPMCs (2002), it is assumed that electrostatic forces, elastic deformation, ion and solvent transport, and osmotic pressure are main driving mechanisms for IPMC response under the applied potential. Different types of solvents have been studied so far to observe various phenomena during IPMC actuation [Nemat-Nasser and Zamani, 2003]. Another way to observe, study, and control different phases of actuation is changing the type of input potential waveform. In what follows, we present the results of the response of a Nafionbased IPMC in $\mathrm{K}^{+}$-form and with ethylene glycol upon various types of input potential waveforms.

Smart Structures and Materials 2004: Electroactive Polymer Actuators and Devices (EAPAD), edited by Yoseph Bar-Cohen, proceedings of SPIE Vol. 5385 (SPIE, Bellingham, WA, 2004)

0277-786X/04/\$15 . doi: 10.1117/12.539075 


\section{IPMC microstructure and properties}

\subsection{Composition and properties}

An IPMC consists of a perfluorinated backbone ionomer (usually Nafion ${ }^{\circledR}$ or Flemion ${ }^{\circledR}$ ) plated on both faces with noble metals (See Figure 1-A) such as platinum or platinum and gold, or gold, and neutralized with a certain amount of counterions that balance the electric charge of the anions covalently bonded to the ionomer. Nafion is a perfluorinated copolymer of polytetrafluoroetheylene with perfluorinated vinyl ether sulfonate pendants [See HeitnerWirguin, 1996 for further information on Nafion backbone ionomer]. Chemical structures of Nafion is shown in Figure 1-B.

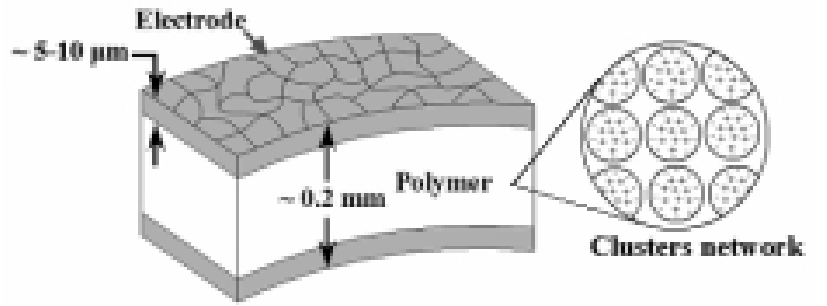

FIG. 1-A. Schematic of an IPMC material

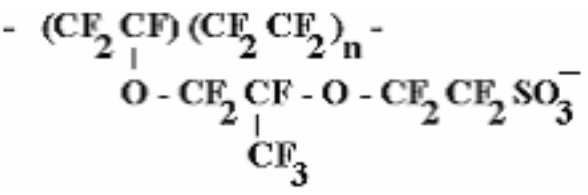

FIG. 1-B. Chemical structure of Nafion

The surface of ionomer is chemically plated with noble metals such as platinum or gold or platinum and a finishing layer of gold. Electrodes in Nafion-based IPMCs consist of 3-10nm diameter metal (generally platinum) particles, distributed mainly within a $10-20 \mu \mathrm{m}$ depth of both faces of the membrane, and usually covered with about $1 \mu \mathrm{m}$ thick gold plating to improve surface conductivity; see Figure 2.

Solfonate groups in Nafion are neutralized with monovalent cations such as alkali metals. For neutralizing counterions, we have used, $\mathrm{Li}^{+}, \mathrm{Na}^{+}, \mathrm{K}^{+}$, and $\mathrm{Rb}^{+}$. The properties of the bare ionomer, as well as that of the corresponding IPMC, change with the cation type for the same membrane.

Dry Nafion-based IPMCs do not show actuation upon application of electric potential. Thus the IPMC samples are fully solvated before stimulation by an electric potential. Most of the hydrated IPMCs electromechanical response studies, so far, are performed in a water bath. This insures that the IPMC sample is fully hydrated during actuation. The initial actuation of IPMCs with alkali metals in a water bath under step DC potential is fast. This initial actuation towards the anode with most alkali metals happens in a fraction of a second. This makes it difficult to study potential mechanisms responsible for IPMCs electrical-chemical-mechanical behavior. Also with water as the solvent, the applied electric potential must be limited to less than $1.3 \mathrm{~V}$ at room temperature, to avoid electrolysis. Moreover, fully hydrated IPMC dries fast in open air. These and related factors limit the study and application of IPMCs with water as the solvent. Other polar solvents, which not only reduce the speed of actuation but also enable us to actuate the IPMCs in open air have been considered. Ethylene glycol or 1,2-Ethanediol $\left(\mathrm{C}_{2} \mathrm{H}_{6} \mathrm{O}_{2}\right)$ is an organic polar solvent, which can be used over a wide range of temperatures.

\subsection{Electromechanical actuation}

The electrical-chemical-mechanical response of the IPMCs depends on the neutralizing cation, the nature and the degree of solvent saturation, the electrode morphology, and the chemical structure of the ionomer backbone. Experiments have been performed to determine the nature of IPMC behavior in response to an applied electric potential [Nemat-Nasser and Zamani (2003) and Nemat-Nasser and Wu (2003)]. When a strip of solvated Nafionbased IPMC sample is subjected to an electric potential of several volts (1-3 V) across its faces, it bends towards the anode. The speed and magnitude of this actuation towards the anode depends on the type of solvent. The actuation towards the anode is relatively slow with ethylene glycol comparing to water, and it is comparatively much slower with glycerol than with water or ethylene glycol as solvents. For Nafion-based IPMCs with alkali metals as counterions, the actuation towards the anode is followed by a slow back relaxation towards the cathode (see Figure 3). The back relaxation speed also depends on the type of solvent. The duration of the back relaxation phase can vary, from less than about 60 seconds (e.g., with most alkali metals and with water), to about 300 seconds (e.g., in $\mathrm{K}^{+}$-form 
with ethylene glycol), and to about 2000 seconds (e.g., in $\mathrm{Na}^{+}$-form with glycerol). The sample eventually reaches an equilibrium state (while the electric potential is still on), which is generally different from its initial equilibrium position. If the electric potential is removed as the two electrodes are shorted, the Nafion-based IPMC sample displays a fast bending deformation towards the cathode and then slowly relaxes back towards the anode, seldom attaining its initial state. Figure 3 illustrates these different phases of actuation for a Nafion-based IPMC in $\mathrm{Na}^{+}$-form with glycerol as the solvent.

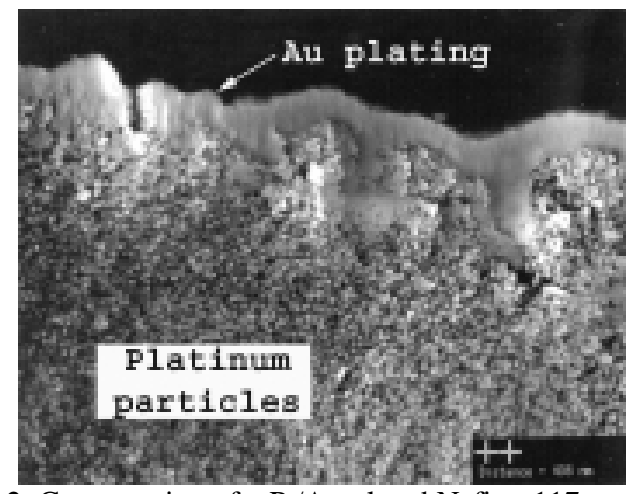

FIG. 2. Cross section of a Pt/Au-plated Nafion-117 membrane at electrode region; distance between crosses is $408 \mathrm{~nm}$

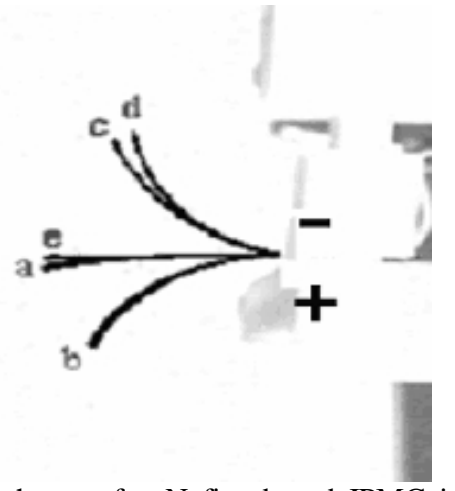

FIG. 3. Different phases of a Nafion-based IPMC in $\mathrm{Na}^{+}$-form with glycerol as solvent, under a $2 \mathrm{~V}$ electric potential (DC): from a to $b$, initial motion towards the anode; from $b$ to $c$, relaxation towards the cathode; from c to d, motion upon shorting; and from $\mathrm{d}$ to e, back towards the anode to equilibrium state

\section{Preparation of cation incorporated IPMCs}

To prepare the samples for tests, first, the as received IPMC sheet is cut by a blade into rectangular pieces of about 2.7 $\mathrm{cm} \times 0.26 \mathrm{~cm}$ using a special jig. The thickness of the Nafion-based composites, in dry state, is about $180 \mu \mathrm{m}$. The procedure to neutralize the sample with different cations is discussed in earlier work [Nemat-Nasser and Zamani, 2003].

\section{Results and discussion}

A typical fully solvated Nafion-based IPMC with most alkali metals under a sustained suddenly applied electric potential (DC), bends towards the anode. The speed and amount of this bending depends on the type of cation, solvent, and degree of solvation. The initial bending towards the anode is followed by back relaxation towards the cathode. Initial actuation towards the anode is assumed to be as a result of the movement of cations towards the anode upon application of a DC potential. Fast motion of cations towards the cathode under step DC potential causes fast initial actuation of IPMC towards the anode [Nemat-Nasser, 2002]. The cluster size in the cathode boundary layer initially increases as a result of cation migration. Nemat-Nasser believes that due to high acidity of sulfonate groups, in time, reorientation of cations occurs in the cathode boundary layer clusters [Nemat-Nasser, 2002]. This is believed to be the reason of the back relaxation followed by osmotic solvent flow. Therefore, if the time needed for cation redistribution is less than the time that the cations move towards the cathode, then we expect that the initial actuation towards the anode would not be observed. To observe this, the potential is increased gradually. The potential is increased linearly to a constant potential (increasing ramp electric potential). Experimental tests on Nafion-based IPMCs with $\mathrm{K}^{+}$-form and with ethylene glycol as solvent under various slopes for the increasing ramp potential are performed. As the potential is applied gradually (smaller slope), the initial actuation towards the anode diminishes. This type of the test also can be used to determine the relaxation time for cation redistribution in the cathode boundary layer. Figure 4 shows an increasing ramp potential of $1.5 \mathrm{~V}$ with the slope of 0.083 . The corresponding IPMC response (normalized curvature) is shown in Figure 5. Normalized curvature (L/R) is defined as the length of the IPMC sample divided by the deformed sample radius of curvature. 


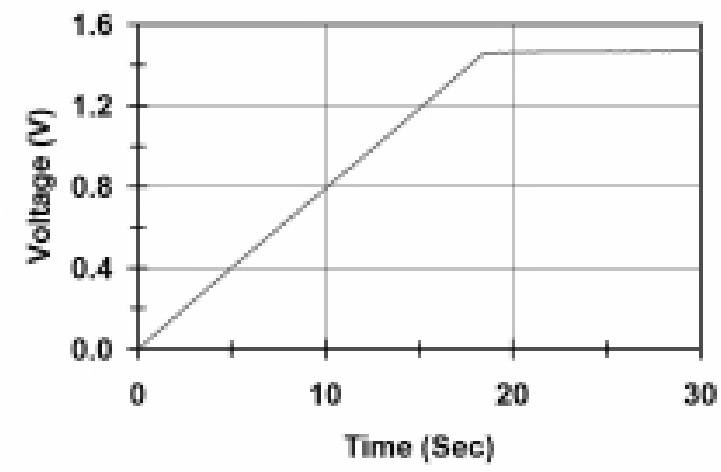

FIG 4. Variation of voltage and current over time

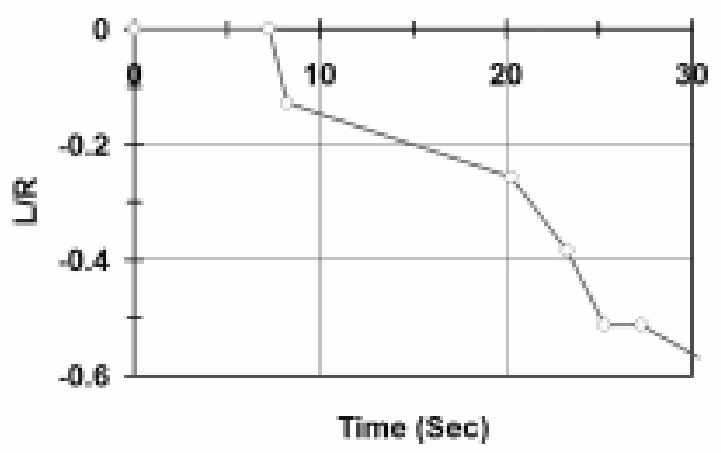

FIG 5. Actuation of a Nafion-based IPMC in $\mathrm{K}^{+}$-form with ethylene glycol as solvent under increasing ramp potential corresponding to Figure 4

In another series of tests, the actuation of a Nafion-based IPMCs under sinusoidal waveform input potential is studied. The actuation of an IPMC strip follows the frequency of the input potential. The excited frequencies are limited to few tens of Hertz. Too low frequencies forces the sample to show back relaxation before the peaks of the input potential is reached. Thus in that case the sample does not follow the input potential. The range of frequencies considered is between 0.05 and $0.2 \mathrm{HZ}$. Figure 6 shows a sinusoidal potential of $1.5 \mathrm{~V}$ with frequency of $0.2 \mathrm{HZ}$. The corresponding IPMC response (normalized tip displacement) is shown in Figure 7. Normalized tip displacement (U/L) is defined as the IPMC tip displacement divided by the length of the IPMC sample.

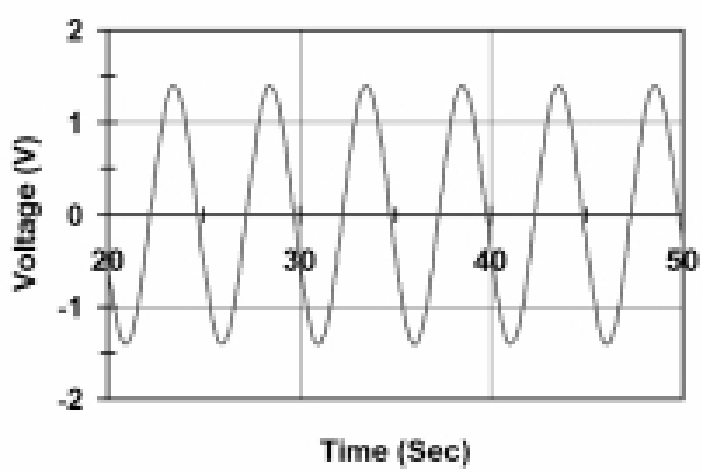

FIG 6. Variation of voltage over time

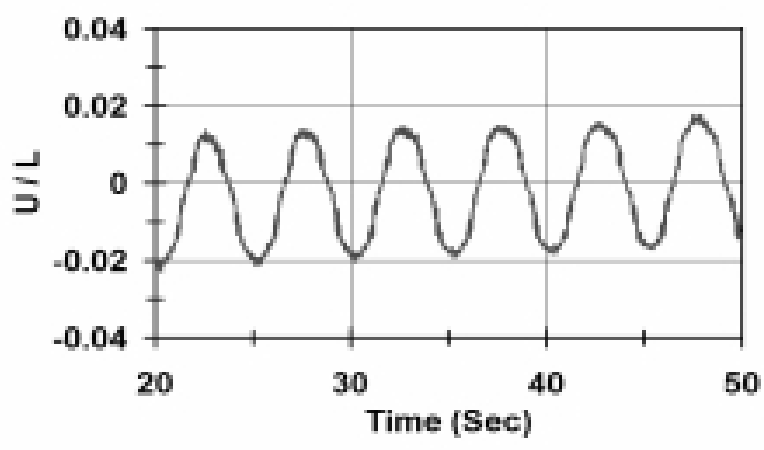

FIG 7. Variation of normalized tip displacement for a Nafionbased IPMC in $\mathrm{K}^{+}$-form with ethylene glycol as solvent under sinusoidal waveform potential corresponding to Figure 6

\section{Conclusions}

Actuation of Nafion-based IPMCs consists of several phases. Initial relatively fast actuation of Nafion-based IPMCs, in most alkali forms is due to migration of cations from the anode to the cathode side. This migration creates the anode and the cathode boundary layers. Controlling the formation speed and behavior of these boundary layers can be achieved through different electric potential waveforms. Using a gradually increasing electric potential can decrease the amount of actuation towards the anode or diminish it completely. This depends on the slope of increasing ramp potential. Using other types of electric potentials such as sinusoidal potential waveform shows that IPMC undergoes the bending vibration at the frequency of the applied potential, generally no more than a few tens of Hertz. 


\section{Acknowledgements}

We wish to thank Drs. Steve Wax (DARPA), Len Buckley (NRL), Carlos Sanday (NRL), and Randy Sands for their continued encouragement and many stimulating discussions; Professor Mohsen Shahinpoor and Dr. K. J. Kim for providing the Nafion-based IPMC samples; Dr. K. Asaka for providing the Flemion-based IPMC samples; Dr. Yosi Bar-Cohen for his continued interest in simulating interaction; Professor Yitzhak Tor for comments and guidance; Mr. Jon Isaacs and Mr. Dave Lischer for developing the experimental equipment and techniques; and Jeff McGee for helpful code to obtain the effective capacitance. This work has been supported by DARPA grant number MDA972-001-0004 to the University of California, San Diego.

\section{References}

[1] Heitner-Wirguin, C., "Recent advances in perfluorinated ionomer membranes: structure, properties and applications," Journal of Membrane Science, 120, 1-33, 1996.

[2] Nemat-Nasser, S., "Micromechanics of actuation of ionic polymer-metal composites," Journal of Applied Physics, 92, 2899-2915, 2002.

[3] Nemat-Nasser, S., and Thomas, C., "Ionomeric polymer-metal composites," in Electroactive Polymer (EAP) Actuators as Artificial Muscles - Reality, Potential and Challenges, edited by Bar-Cohen, SPIE, Bellingham, WA, 2001, Chap. 6, pp. 139-191.

[4] Nemat-Nasser, S., and Wu, Y., "Comparative experimental study of ionic polymer-metal composites with different ionomers and in various cation forms," Journal of Applied Physics, 93, No. 9,2003.

[5] Nemat-Nasser, S., and Zamani, S., "Experimental Study of Nafion- and Flemion-based Ionic Polymer-metal Composites (IPMCs) with Ethylene Glycol as Solvent," Elecctroactive Polymer Actuators and Devices, edited by Y.Bar-Cohen, Smart Structures and Materials 2003, Proceedings of SPIE Press, 5051, 233-244, 2003.

[6] Onishi, K., Sewa, S., Asaka, K., Fujiwara, N., and Oguro, K., "Morphology of electrodes and bending response of the polymer electrolyte actuator," Electrochemica Acta, 46, 737-743, 2000.

[7] Onishi, K., Sewa, S., Asaka, K., Fujiwara, N., and Oguro, K., "The effects of counter ions on characterization and performance of a solid polymer electrolyte actuator," Electrochemica Acta, 46, 1233-1241, 2001. 